\title{
Flat Spectrum Radio Quasar Evolution and the Gamma-ray Background
}

\author{
Jack Singal $^{1}$, Allan $\mathrm{Ko}^{2}$ and Vahe Petrosian ${ }^{3}$ \\ ${ }^{1}$ University of Richmond \\ Physics Department \\ 28 Westhampton Way, Richmond, VA 23173 \\ email: jsingal@richmond.edu \\ ${ }^{2}$ Department of Physics, Masacusetts Institute of Technology \\ 77 Massachusetts Avenue, Cambridge, MA 02139-4307 \\ ${ }^{3}$ Kavli Institute for Particle Astrophysics and Cosmology \\ Departments of Physics and Applied Physics, Stanford University \\ 382 Via Pueblo Mall, Stanford, CA 94305-4060
}

\begin{abstract}
We discuss an analysis of the redshift evolutions and distributions of the gamma-ray luminosity and photon spectral index of flat spectrum radio quasar (FSRQ) type blazars. We utilize data from the Fermi Gamma-ray Space Telescope, with redshfits as determined from optical spectroscopy by Shaw et al. We find that FSRQs have evolved significantly in luminosity but negligibly in photon index, and contribute in toto roughly $20 \%$ of the total gamma-ray output of the Universe.
\end{abstract}

\section{Introduction}

Blazars comprise the vast majority of the extragalactic objects in the gamma-ray sky seen by instruments including the Fermi Gamma-ray Space Telescope, as discussed in e.g. Abdo et al. (2010a). Blazars are active galactic nuclei (AGNs) for which the jet is aligned with our line of sight, and are unique among AGN classes in commonly featuring prominent gamma-ray emission. They are a natural candidate for an important source population of the extragalactic gamma-ray background (EGB) radiation.

Blazars are customarily divided into two classes based on their optical emission properties - FSRQs which feature prominent and broad optical emission lines, and BL Lacertae objects (BL Lacs) which do not. Shaw et al. (2012) provide spectroscopically-determined redshifts for almost all of the FSRQ blazars from the Fermi-Large Area Telescope (LAT) first year catalog. With these redshifts, one has a dataset of FSRQs with known luminosities that is limited only by the gamma-ray observations. Given such a dataset, we can apply techniques that we have developed to determine the evolutions of the luminosity and photon index with redshift, the density evolution, and the distributions of luminosity and photon index for FSRQs non-parametrically and directly from the gamma-ray and redshift data. With these evolutions and distributions, we can calculate the total gamma-ray output of FSRQs over the history of the Universe, and compare it to the EGB. The analysis summarized here is more thoroughly discussed in Singal et al. (2014).

\section{Data and Methods}

The blazars from the first-year Fermi-LAT extragalactic catalog, which was presented in Abdo et al. (2010b), are shown in Figure 1. The flux shown is the integrated photon flux between $100 \mathrm{MeV}$ and $100 \mathrm{GeV}$, and is denoted $F_{100}$. Because of the energy dependence 
of the Fermi-LAT point spread function, hard spectrum objects can be seen to lower fluxes than soft spectrum objects. This leads to the data for blazars being truncated in the flux-index plane, as can be seen in Figure 1.

We have developed techniques to recover the intrinsic distributions of population characteristics non-parametrically and directly from such truncated data. These methods are based on the analyses of Efron and Petrosian in works such as Efron \& Petrosian (1992) and Efron \& Petrosian (1999). In recent years we have extended and applied these methods to multi-dimensional distributions including the radio and optical luminosity evolution of quasars in Singal et al. (2011) and Singal et al. (2013), the flux and photon index distribution of blazars in Singal et al. (2012), and the X-ray luminosity and plateau end time distributions of gamma-ray bursts in Dainotti et al. (2013).

The methods involve the use of "associated sets" of data for comparison. A version of the Kendall Tau test modified with associated sets is implemented to determine the true intrinsic correlations among variables, such as luminosity and redshift, in a truncated data set. To compute distributions such as luminosity functions with truncated data, we use the method of Lynden-Bell (1971), which is equivalent to a maximum likelihood determination, but modified with the use of associated sets. In addition to being implemented in the works mentioned above, these methods have been verified with simulated data sets, and detailed discussions of them are available in e.g. Singal et al. (2013) and Singal et al. (2014).

Here we discuss the application to Fermi-LAT FSRQs with redshifts. We parameterize a general luminosity function (LF), with luminosity and density evolution, in a waveband $a$ as

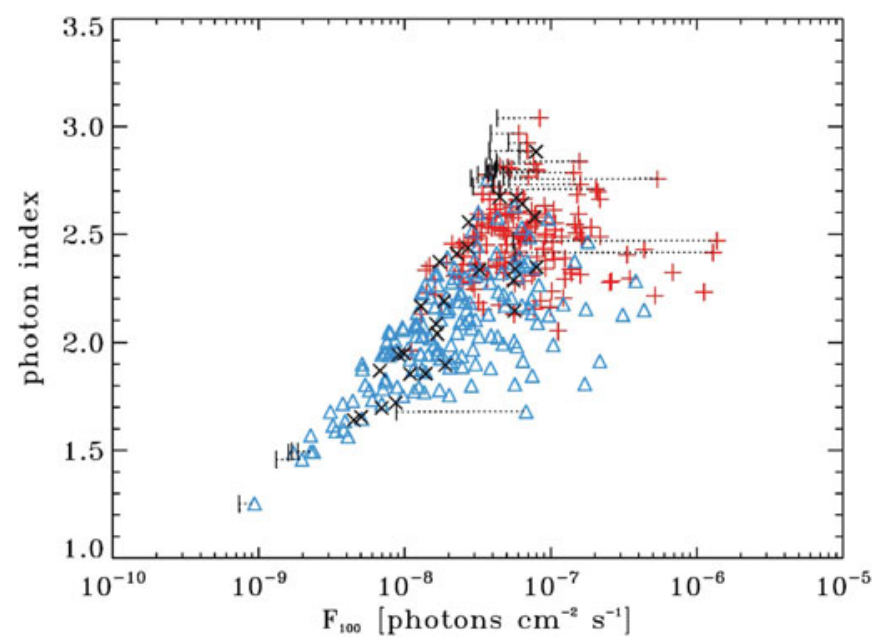

Figure 1. Flux and photon spectral index for the 352 blazars from the Fermi-LAT first year catalog with detection test statistic $\geqslant 50$ and $|b| \geqslant 20^{\circ}$. FSRQs type blazars $(n=161)$ are shown as red plus signs, BL Lac type blazars $(n=163)$ are shown as blue triangles, and blazars of unidentified or ambiguous type $(\mathrm{n}=28)$ are represented by black x's. A subsequent analysis by Shaw et al. (2012) determined that approximately 20 of those classified as either unknown or BL Lacs were actually FSRQs, and we have included these in our analysis. It is seen that there is a selection bias against soft spectrum sources at fluxes below $\sim 10^{-7}$ photons $\mathrm{cm}^{-2} \mathrm{sec}^{-1}$. We also show for a selection of sources (but only a few for clarity) the approximate limiting flux for that source - that is the lowest flux it could have and still be sufficiently bright to be included in the sample given its location on the sky given the reported detection significance. 


$$
\Psi_{a}\left(L_{a}, z\right)=\rho(z) \psi_{a}\left(L_{a} / g_{a}(z)\right) / g_{a}(z)
$$

where $g_{a}(z)$ describe the luminosity evolution with redshift and $\rho(z)$ describes the comoving density evolution with redshift. Once the luminosity evolution factor $g_{a}(z)$ is determined by quantifying the correlation between the luminosity and redshift, we can obtain the mono-variate distributions of the independent variables $L_{\mathrm{a}}^{\prime}=L_{\mathrm{a}} / g_{\mathrm{a}}(z)$ and $z$. Those distributions are the density evolution $\rho(z)$ and "local" LF $\psi_{\mathrm{a}}$. We consider this form of the LF for the gamma-ray luminosity, and a similar form for the photon index distribution.

Because the sample at hand consists of relatively low redshift objects, we assume a simple power law for the evolutions within this redshift range

$$
g_{\mathrm{a}}(z)=(1+z)^{k_{\mathrm{a}}} .
$$

The determination of the luminosity evolution and photon index evolution then reduce to the determination of $k_{\mathrm{L}}$ and $k_{\Gamma}$, which are themselves simply the best-fit power-law correlation between luminosity and redshift, and index and redshift, respectively. We determine these best-fit correlations with the modified Kendall Tau test with associated sets mentioned above, further modified to accommodate two dependent variables (luminosity and index).

Once the evolutions and distributions have been determined, the integrated gammaray output of all FSRQs between $100 \mathrm{MeV}$ and $100 \mathrm{GeV}$ can be calculated by performing an integration of the distributions over redshift, luminosity, and index. As discussed in Singal et al. (2014), the resulting integral can be reduced to

$\mathcal{I}_{\gamma: \mathrm{FSRQs}}=\int_{z} d z \int_{\Gamma=-\infty}^{\infty} d \Gamma \int_{L_{\gamma}=0}^{\infty} d L_{\gamma} \frac{1}{4 \pi D_{L}^{2} K_{L}(z, \Gamma) g_{\mathrm{L}}(z)} \rho(z) \frac{d V}{d z} \Phi_{\mathrm{L}_{\gamma}^{\prime}}\left(\frac{L_{\gamma}}{g_{\mathrm{L}_{\gamma}}(z)}, z\right) h(\Gamma)$

where $\Phi_{L_{\gamma}^{\prime}}$ is the local cumulative luminosity function and $h(\Gamma)$ is the index distribution. This allows us to calculate the total integrated output of FSRQs by using the direct

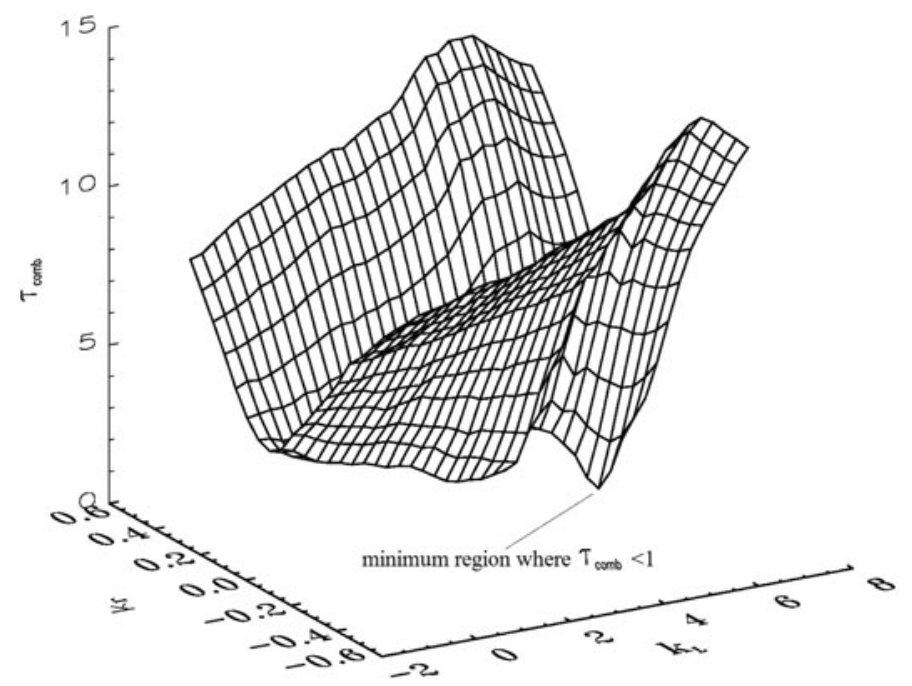

Figure 2. Surface plot of the value of the test statistic $\tau_{\text {comb }}$ showing the location of the minimum region where the favored values of the redshift evolution power law factors $k_{\mathrm{L}}$ and $k_{\Gamma}$ lie, for the forms of the evolutions given by equation 2.2 . 
output of our analysis methods, the luminosity and density distributions, without any fitting.

\section{Results and Implications}

We find that FSRQs exhibit strong luminosity evolution, but negligible photon index evolution, with redshift. Figure 2 shows a surface plot of the goodness-of-fit statistic $\tau_{\text {comb }}$ resulting from the modified multi-dimensional Kendal tau test for the simultaneous determination of the best-fit $k_{\mathrm{L}}$ and $k_{\Gamma}$, clearly showing the preferred values of a significantly nonzero and positive $k_{\mathrm{L}}$ and a negligible $k_{\Gamma}$. We also find that FSRQs evolve in density strongly with the comoving differential density evolution peaking at redshift $z \sim 1$.

Performing the integration of equation 2.3 we find that FSRQs have a total output from $100 \mathrm{MeV}$ to $100 \mathrm{GeV}$ over the history of the Universe of $\mathcal{I}_{\gamma: \text { FSRQs }}=1.0(+0.4 /-$ 0.1) $\mathrm{MeV} \mathrm{cm}^{-2} \mathrm{sec}^{-1} \mathrm{sr}^{-1}$. This can be compared to the total EGB in this photon energy range, resolved and unresolved, as measured by the Fermi-LAT, to reveal that FSRQs contribute $17(+10 /-4) \%$ of the total EGB. The results as presented here are in reasonable agreement with those presented in Ajello et al. (2012), which are arrived at using the same data set but very different methods. In that work they report that FSRQs contribute $21.7(+2.5 /-1.7) \%$ of the total EGB.

The strong intrinsic positive correlation of gamma-ray luminosity with redshift, along with positive evolutions in other wavebands such as optical and radio as seen in e.g. Singal et al. (2013), and X-ray as seen in e.g. Aird et al. (2010), favors models in which at higher redshifts AGN systems had on average more massive black hole and accretion disk systems, and/or black holes with larger spin parameters. The lack of appreciable photon index evolution with redshift in FSRQs indicates that the mean spectrum of accelerated high energy particles from their central engines has remained largely constant over the history of the Universe. The result that FSRQs are a significant but not dominant contributor to the EGB implies that other source classes such as BL Lacs, and possibly starforming galaxies as discussed in e.g. Lacki et al. (2011), and non-blazar AGN as discussed in e.g. Inoue \& Totani (2011), are important. The data, methods, and results summarized here are discussed in further detail in Singal et al. (2014).

\section{References}

Abdo, A., et al. 2010a, ApJS, 188, 405

Abdo, A., et al. 2010b, ApJ, 715, 429

Ajello, M., Shaw, M., Romani, R., Dermer, C., Costamonte, L., King, G., \& Max-Moerbeck, W. 2012, ApJ, 751, 108

Aird, J., et al. 2010, MNRAS, 401, 2531

Dainotti, M., Petrosian, V., Singal, J., \& Ostrowski, M. 2013, ApJ, 774, 157

Efron, B. \& Petrosian, V. 1992, ApJ, 399, 345

Efron, B. \& Petrosian, V. 1999, JASA, 94, 447

Inoue, Y. \& Totani, T. 2011, ApJ, 728, 73

Lacki, B., Thompson, T., Quataert, E., Loeb, A., \& Waxman, E. 2011, ApJ, 734, 107

Lynden-Bell, D. 1971, MNRAS, 155, 95

Shaw, M., et al., 2012, ApJ, 748, 49

Singal, J., Petrosian, V., J., Lawrence, A., \& Stawarz, Ł. 2011, ApJ, 743, 104

Singal, J., Petrosian, V., \& Ajello, M. 2012, ApJ, 753, 45

Singal, J., Petrosian, V., Stawarz, Ł., \& Lawrence, A. 2013, ApJ, 764, 43

Singal, J., Ko, A., \& Petrosian, V. 2014, ApJ, 786, 109 